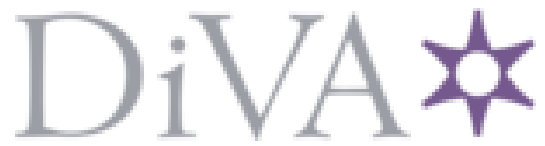

http://www.diva-portal.org

Preprint

This is the submitted version of a paper published in Advanced Functional Materials.

Citation for the original published paper (version of record):

Granskog, V., García-Gallego, S., von Kieseritzky, J., Rosendahl, J., Stenlund, P. et al. (2018)

High-Performance Thiol-Ene Composites Unveil a New Era of Adhesives Suited for Bone Repair

Advanced Functional Materials, 28(26): 1800372

https://doi.org/10.1002/adfm.201800372

Access to the published version may require subscription.

N.B. When citing this work, cite the original published paper.

Permanent link to this version:

http://urn.kb.se/resolve?urn=urn:nbn:se:ri:diva-33958 


\title{
High-Performance Thiol-Ene Composites Unveil a New Era of Adhesives Suited for Bone Repair
}

\author{
Viktor Granskog, Sandra García-Gallego, Johanna von Kieseritzky, Jennifer Rosendahl, \\ Patrik Stenlund, Yuning Zhang, Sarunas Petronis, Benny Lyvén, Marianne Arner, \\ Joakim Håkansson, and Michael Malkoch**
}

The use of adhesives for fracture fixation can revolutionize the surgical procedures toward more personalized bone repairs. However, there are still no commercially available adhesive solutions mainly due to the lack of biocompatibility, poor adhesive strength, or inadequate fixation protocols. Here, a surgically realizable adhesive system capitalizing on visible light thiol-ene coupling chemistry is presented. The adhesives are carefully designed and formulated from a novel class of chemical constituents influenced by dental resin composites and self-etch primers. Validation of the adhesive strength is conducted on wet bone substrates and accomplished via fiber-reinforced adhesive patch (FRAP) methodology. The results unravel, for the first time, on the promise of a thiol-ene adhesive with an unprecedented shear bond strength of $9.0 \mathrm{MPa}$ and that surpasses, by $55 \%$, the commercially available acrylate dental adhesive system Clearfil SE Bond of $5.8 \mathrm{MPa}$. Preclinical validation of FRAPs on rat femur fracture models details good adhesion to the bone throughout the healing process, and are found biocompatible not giving rise to any inflammatory response. Remarkably, the FRAPs are found to withstand loads up to $70 \mathrm{~N}$ for 1000 cycles on porcine metacarpal fractures outperforming clinically used K-wires and match metal plates and screw implants.

vehicles. ${ }^{[1]}$ The thiol-ene coupling (TEC) reaction is persistent under various conditions including the presence of water and oxygen. As a result, TEC chemistry is of high interest for in situ reactions in biomedical applications such as hydrogels, ${ }^{[2]}$ tooth fillings, ${ }^{[3]}$ and tissue adhesives. ${ }^{[4]}$ The stepwise polymerization of thiol-ene networks promotes a homogenous structural buildup that is highly advantageous when mechanical robustness is a prerequisite. Recently, TEC chemistry has been evaluated in the dental field. Attempts to substitute methacrylate composite resins with thiol-ene replacements have been assessed in order to overcome mechanical stress generated during the curing process and to generate restorations with lower leakage of monomers that can cause toxicity. ${ }^{[3]}$ The TEC chemistry has also been surveyed for developing biocompatible adhesives for bone fracture fixation. ${ }^{[4 b-d]}$

\section{Introduction}

The chemical reaction between sulfuric compounds and unsaturated double bonds has displayed its undenied success over the years and has been advanced from vulcanization of rubbers to delicate lithography and drug delivery

V. Granskog, Dr. S. García-Gallego, Dr. Y. Zhang, Prof. M. Malkoch KTH Royal Institute of Technology

Department of Fibre and Polymer Technology

SE-100 44 Stockholm, Sweden

E-mail:malkoch@kth.se

Dr. J. von Kieseritzky, Prof. M. Arner

Department of Clinical Science and Education

and the Department of Hand Surgery

Karolinska Institutet

SE-118 83 Stockholm, Sweden

J. Rosendahl, Dr. P. Stenlund, Dr. S. Petronis, Dr. B. Lyvén,

Prof. J. Håkansson

RISE Research Institutes of Sweden

Bioscience and Materials-Medical Device Technology

Box 857 Borås, Sweden

The ORCID identification number(s) for the author(s) of this article can be found under https://doi.org/10.1002/adfm.201800372.

DOI: 10.1002/adfm.201800372
However, despite ambitious attempts to introduce TEC chemistry to generate adhesives for hard tissue restoration, the final performance of the TEC adhesives has been insufficient to match the high demands of the field and in parallel failing to reach the performances present for commercially available acrylate dental systems. Nevertheless, with the lack of adhesives for bone fracture fixations a clear opportunity exists. The use of cyanoacrylates, ${ }^{[5]}$ bone cements, ${ }^{[6]}$ and other explored suggestions such as isocyanate-polyols chemistry $^{[7]}$ or biomimetic strategies using phosphates, amines, and catechols has proven promising, ${ }^{[8]}$ but has not expanded the area to its full potential, where either bond strength, doubtful biocompatibility, and inadequate adhesive methodology have been the main obstacles..$^{[5 c, 6 c, 9]}$ For thiol-ene adhesives, the lack of mechanical rigidity and adhesion to wet surfaces to promote a high bond strength has been challenges to overcome until now.

Here, we report on a combined design strategy for high bond strength and its exploitation for fracture repair that is inspired by the technology used in dental restorations and translated to fit thiol-ene resins to unlock a new era of new high-performance biomedical TEC adhesives with unmet biocompatibility. 


\section{Results and Discussion}

\subsection{TEC Resin Composite}

Dental adhesives are scarcely represented when biomedical adhesives are discussed in the scientific literature ${ }^{[10]}$ despite their preeminent adhesion to bony tissues. Among them, self-etch systems containing acrylate based monomers with acidic adhesion-enhancing groups such as phosphoric, phosphonic, or carboxylic acids display promising attributes for bone adhesion. ${ }^{[6 a, 11]}$ The acidic monomers can, on the one hand, dissolve hydroxyapatite and expose the collagen fibers, and consequently enable entanglemental surface binding. On the other hand, the acidic monomers can create ionic bonds to calcium ions forming precipitates with very low solubility that integrate into the bone surface. Dental adhesive's rigidity and load bearing properties are obtained through a subsequent addition of resin composite mainly consisting of glass fillers. These adhesives have displayed great success in dental restorations over the years and may be of interest as bone adhesives; however, the use of acrylate polymerization systems is typically coupled to leakage of harmful residues, such as unreacted monomers ${ }^{[9 e, 12]}$ and bisphenol A. ${ }^{[13]}$ Even though the levels of residue released are not considered toxic, the differences in material amount and oral versus parenteral exposure need to be considered comparing dental and bone restorations. With TEC chemistry the leakage of harmful residues can be minimized by designing mild components and through the high-conversion crosslinking that reduces the release of low molecular weight compounds from the adhesive and increasing the safety of the product.

Building on the inspiration from dental resin composites, TEC composites based on a two-component adhesive of tris[2(3-mercapto propionyloxy)ethyl] isocyanurate (TEMPIC) and 1,3,5-triallyl-1,3,5-triazine-2,4,6 $(1 \mathrm{H}, 3 \mathrm{H}, 5 \mathrm{H})$-trione (TATATO) were carefully formulated with hydroxyapatite (HA) particles (Figure 1). An amount of $56 \mathrm{wt} \% \mathrm{HA}$ was found as optimal filler content in the adhesive to secure a surgically manageable viscous liquid and in parallel maintaining high amount of filler to elevate stiffness. The introduction of HA fillers increased the flexural modulus of the adhesive material with 244\%, from 2.5 (s.e.m. 0.5) GPa to 6.1 (s.e.m. 0.8) GPa $(p=0.009$ ) (Figure 1c). Interestingly, the use of high energy visible (HEV) light and a change from the well-known high-wavelength photoinitiator camphorquinone to Irgacure 819 enabled sufficient curing depth for TEC adhesive applications despite a white and opaque appearance of the adhesive-HA mixture. High conversion of functional groups was obtained with only $10 \mathrm{~s}$ of HEV-light irradiation, displayed through analysis of FT-Raman spectroscopy (Figure 1d).

\subsection{TEC Self-Etching Primer}

To continue in the path of dental resin inspiration to self-etch primers for high bond strength, two dual purpose adhesionenhancing molecules were derived from 3-(allyloxy)-2-((allyloxy)methyl)-2-methylpropanoic acid (BAPA) to contain phosphonic acid (Phn) moieties as bone-adhesive groups and alkene
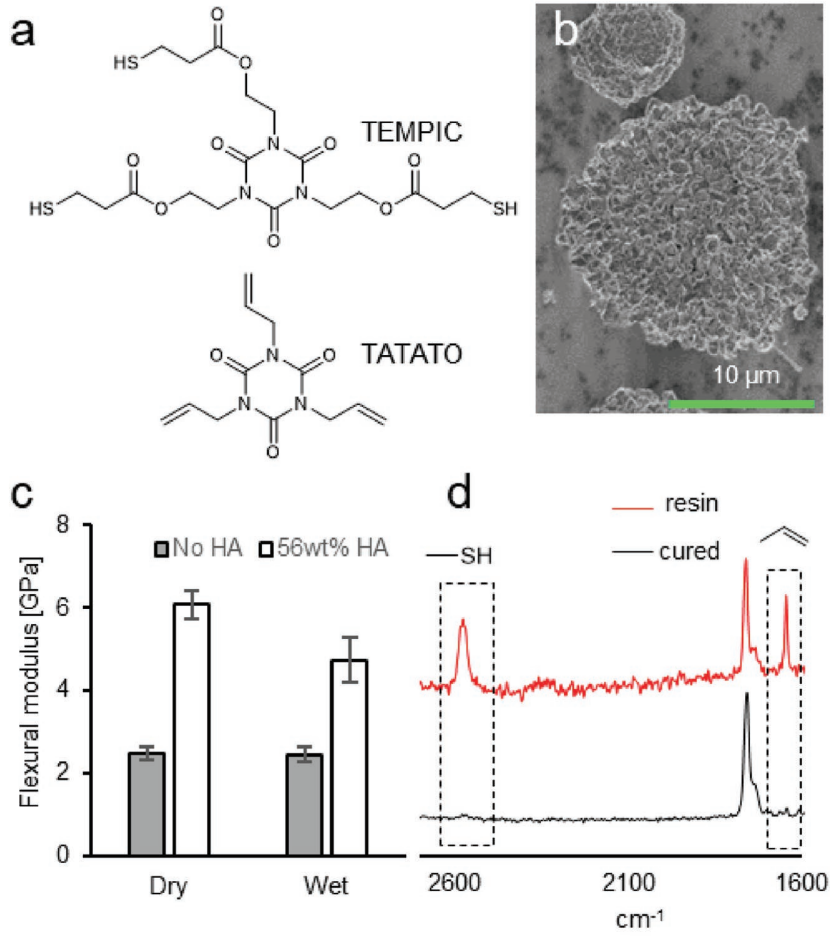

Figure 1. a) The matrix components and b) the HA particle fillers of the adhesive composite. c) Flexural modulus of the adhesive w/w HA filler. d) FT-Raman spectroscopy of the thiol-ene resin (red) and the TEC composite after $10 \mathrm{~s}$ of curing (black).

moieties available for TEC chemistry (Figure 2a; Figure S1, Supporting Information). The model compounds ((3-(allyloxy)2-((allyloxy)methyl)-2-methylpropanamido)methyl)phosphonic acid (BAPA $\left.{ }^{\mathrm{Phn}}\right)$, ((2-(3-(allyloxy)-2-((allyloxy)methyl)-2 methylpropanamido)ethyl)azanediyl)bis(methylene))bis(phosphonic acid) (BAPA ${ }^{\text {bisPhn }}$, and BAPA together with an interconnecting thiol molecule ethoxylated-trimethylolpropane tri-3-mercaptopropionate (ETTMP) provided the foundation for a thorough examination of a primer composition with superior adhesive strength to wet bone substrates. To achieve TEC-adhesive systems with strong mechanical performance, the polymerization needs to progress properly as well as the primer and adhesive compositions need to be close to stoichiometric ratios. The biocompatible and water-soluble photoinitiator lithium phenyl-2,4,6-trimethylbenzoylphosphinate (LAP) was used in the primer solution to ensure an efficient initiation and polymerization of the primer components. ${ }^{[14]}$

The bond strength to bone substrates was assessed by evaluating different compositions of the TEC-adhesive system as fiber-reinforced adhesive patches (FRAPs), capitalizing on a bottom-up engineering approach via layer-by-layer deposition. FRAPs were prepared on wet bone pieces, crafted from bovine femur, with defined geometries and surface roughness to eliminate any deviations of shapes and surfaces that usually are obtained when natural tissues are used. Each FRAP was accomplished by evenly applying a primer layer on the bone surface followed by a drying procedure using gentle air flow. Subsequently, a medically approved poly(ethylene terephthalate) (PET) fiber mesh embedded in the adhesive 


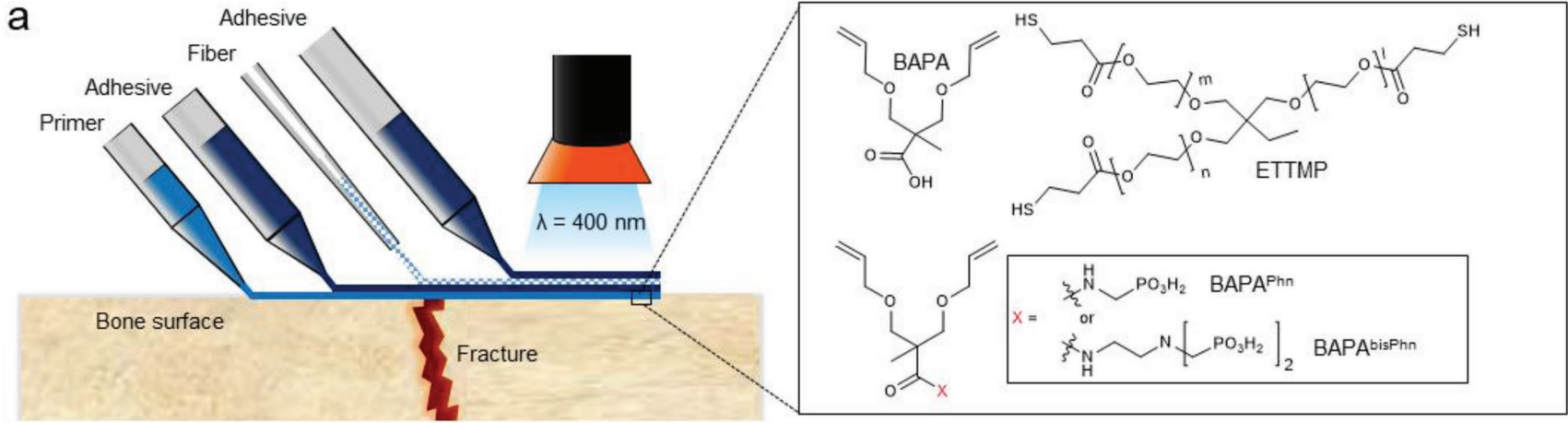

b

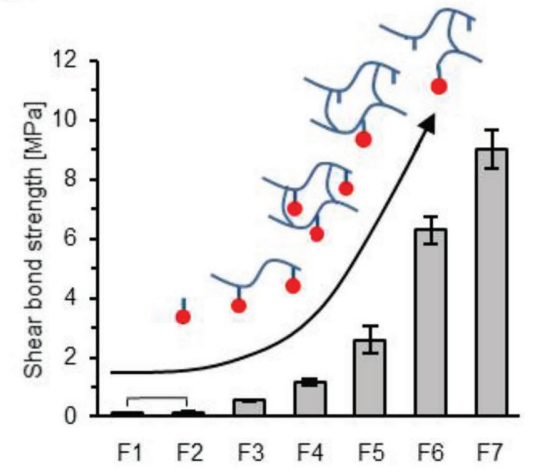

C

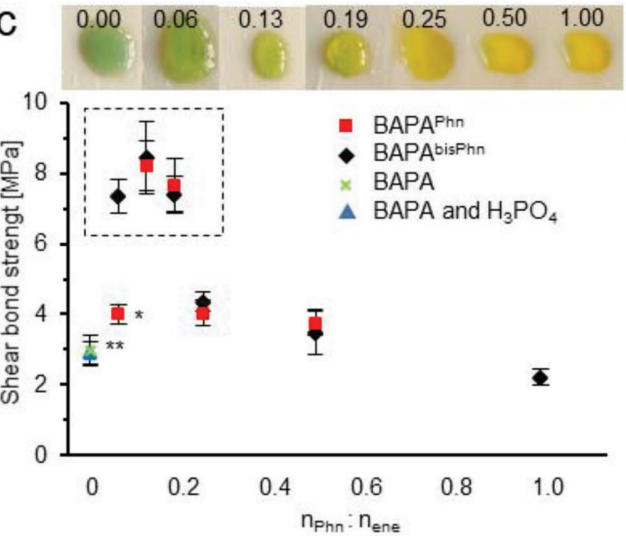

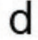

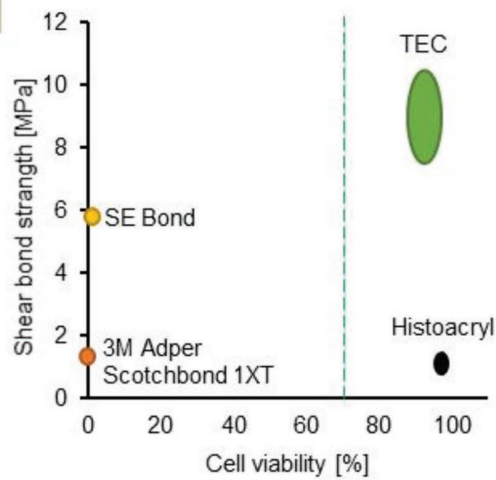

Figure 2. a) A schematic overview of the FRAP build-up and primer components. b) The increase of shear bond strength depending on the molecular linking strategy of FRAP on crafted bone. Bracket displays $p>0.05$ between two populations. $c)$ The ratio between phosphonic acid groups ( $\left.n_{\text {Phn }}\right)$ and ene groups $\left(n_{\text {ene }}\right)$ and a BTB pH indication on bone treated with solutions of BAPA ${ }^{\text {bisphn }}$ corresponding to the $n_{\text {phn }}: n_{\text {ene }}$ ratios and its effect on bond strength. $\mathrm{pH}$ of initial primer solutions was between $2<\mathrm{pH}<3$ except for the two samples $*(\mathrm{pH}=3)$ and $* *(\mathrm{pH}=4)$. The different symbols display which adhesion-enhancing molecule that was used. Compositions with the same $n_{\text {phn }}: n_{\text {ene }}$ ratio have $p>0.05$ except the 0.06 ratio, $p<0.05$. Compositions in dashed box have $p<0.05$ to the other compositions. d) An overview of the shear bond strength compared to cytotoxicity of tested adhesives on murine monocytes. The green dashed line represents the limit where the material is considered toxic. Staples in graphs represent average values and error bars are s.e.m. Two-sided Mann-Whitney U-test was used for statistical analysis.

mixture was applied on the primer layer and effectively cured in seconds via HEV light TEC chemistry to establish the first layer. An iterative addition process with a total of five layers of fiber mesh and adhesive mixtures followed by a top coating of adhesive resulted in a complete FRAP with a smooth surface. While this study focused on five layers of adhesive and fiber mesh, it should be noted that the layer-by-layer strategy is modular with respect to the design of number of adhesive layers and consequently forces the FRAP need to withstand. To mimic realistic conditions, all FRAPs were engineered on bone pieces that were immersed in modified simulated body fluid $(\mathrm{m}-\mathrm{SBF})^{[15]}$ for $24 \mathrm{~h}$ at $37{ }^{\circ} \mathrm{C}$ before the shear bond strength was evaluated. A comprehensive investigation clarified the optimal primer composition, going from single dual-purpose molecule, via in situ chainwise linking to complete network formation of the primer on the bone surface as a connected multisite anchor with the trithiol crosslinker (5) (Figure 2a). The thorough investigation provided valuable insight into optimal primer conditions, suited specifically for TEC systems, with shear bond strength obtained from low $0.16 \mathrm{MPa}$ (s.e.m. 0.04) for single dual-purpose molecule (F2) to high $9.0 \mathrm{MPa}$ (s.e.m. 0.7) for primer network (F7). For the latter, the highest shear bond strength documented for the bone adhesives was capitalized on an off-stoichiometric ratio between thiols and alkenes (OSTE), 1:1.1 thiol:ene. The OSTE strategy, with accessible enes, resulted in an optimal primer network that facilitates subsequent covalent bonding to the adhesive mixture. Furthermore, the study revealed the importance of maintaining a low level of phosphonic acid moieties to achieve high bond strength (Figure 2c). Contrary to the expectations, higher bond strength was obtained at lower amount of phosphonic acid in the primer composition. These findings can be compared to acrylic systems in which phosphonic acid moieties have proved to increase the polymerization rate of acrylic monomers. ${ }^{[16]}$ However, it is known that trivalent phosphorous compounds are radical trapping agents and similar structures to BAPA $^{\text {Phn }}$ and BAPA ${ }^{\text {bisPhn }}$ have been suggested as stabilizer in thiol-ene resins. ${ }^{[17]}$ Since TEC chemistry is a stepwise reaction, a well crosslinked network at the bone-adhesive interface is pivotal. In this context, radical inhibition due to the presence of phosphorous compounds in the primer could have significant effect on the polymerization reaction and consequently the final bond strength of the adhesive. Mixtures of $\mathrm{BAPA}$ and $\mathrm{BAPA}^{\mathrm{Phn}}$ or $\mathrm{BAPA}^{\text {bisphn }}$ displayed the highest shear 
bond strength at a molar ratio of $0.06-0.19$ of phosphonic acid groups in comparison to the TEC functional groups. Moreover, the importance of preserving the appropriate ratio was further corroborated by using half of the number of BAPA ${ }^{\text {bisPhn }}$ molecules in comparison to $\mathrm{BAPA}^{\mathrm{Phn}}$. In this case, $\mathrm{BAPA}^{\text {bishn }}$ induced similar bond strength to BAPA ${ }^{\mathrm{Phn}}$ except at very low amounts of phosphonic acid (Figure 2c). The eventual excess of phosphonic acids at the primed surface was evaluated with aqueous solutions of BAPA ${ }^{\text {bisPhn }}$ corresponding to the $n_{\mathrm{Phn}}: n_{\mathrm{ene}}$ ratios tested. The solutions were applied on bone surfaces and the magnitude of neutralization of adhesion-enhancing phosphonic molecules, through buffering, was assessed with a colorimetric bromothymol blue (BTB) solution. A clear color shift, from acidic yellow ( $\mathrm{pH}$ of $\approx 2$ ) to neutral green ( $\mathrm{pH}$ of $\approx 7$ ), was observed when BTB was applied on bone substrates treated with the different phosphonic solutions. In this context, primer mixtures of $0.06-0.19 n_{\mathrm{Phn}}: n_{\mathrm{ene}}$ ratios with the highest bond strength achieved could be directly correlated to neutral $\mathrm{pH}$ character of the bone substrates. Higher concentration of BAP$\mathrm{A}^{\text {bisphn }}$, i.e., $\geq 0.25 n_{\mathrm{Phn}}: n_{\mathrm{ene}}$ ratios, was found excessive in which all phosphonic groups could not be neutralized by the bone surfaces. These findings can be translated to insufficient release of calcium phosphate from the bone surface for proper integration of BAPA ${ }^{\text {bisphn }}$ and subsequently an excess of phosphonic acid that may inhibit proper crosslinking via TEC chemistry. To exclude any increase of bond strength due to $\mathrm{pH}$ difference of primers, an additional experiment was performed where a primer composition of BAPA and ETTMP with the $\mathrm{pH}$ adjusted with phosphoric acid did not increase shear bond strength values. Importantly, the highest obtained shear bond strength of 9.0 MPa goes beyond the values obtained in FRAPs on wet bone surfaces with the commercial acrylate dental self-etching primer and adhesive system of Clearfil SE Bond of $5.8 \mathrm{MPa}$ (s.e.m. 0.1 ) that failed due to its brittle behavior. The obtained fixation with the thiol-ene adhesive is indeed remarkable with over $55 \%$ increase compared to the dental system, recognized as one of the best adhesives available for dental restorations. A comparative study on adhesive performance and cytocompatibility of the high-performance medical adhesives Adper Scotchbond 1XT, Histoacryl, Clearfil SE Bond, and the TEC FRAP further cement the capability of TEC chemistry in high-performance biomedical adhesives (Figure 2d; Figure S9, Supporting Information).

\subsection{FRAP Biocompatibility}

Satisfied with the superior adhesive performance and initial cytocompatibility of the FRAPs based on TEC chemistry and in comparison to dental adhesives, an elaborate biological study, both in vitro and in vivo, was conducted of the TEC adhesive. A comprehensive safety evaluation (ISO 10993-1:2009) was conducted, in accordance to Good Laboratory Practice (GLP), on FRAP leachables. The study revealed the benign nature of the TEC composition in which no cytotoxicity or genotoxicity was detected nor provoking any skin irritation or inflammation (Figure S10, Supporting Information). In in vivo rat fracture models, the bottom-up buildup of the FRAPs was validated as a realistic procedure by hand surgeons and each operation was successfully completed within 20 min (Figure 3a). The mixing of the adhesive systems was performed before the initiated surgeries and the adhesive and primer were successfully used for at least $4 \mathrm{~h}$ before a change to new batches was made. The stability of the mixed adhesive depends on light exposure and the mixed adhesives have proven stable for over one day in complete darkness. The bone healing was equivalent in both the FRAP group and the reference group (RatFix fixation) after 5 weeks of healing with callus formation around the cortical bone, in the fracture area, outside the cortical bone, and in the medullary cavity (Figure 3b,c; Figures S11-S13, Supporting Information). A thin soft tissue integument was found localized between the FRAP and the cortical bone and no inflammatory responses were detected in any of the groups (Figure $3 \mathrm{~d}$ ). In addition, a pre and post in vivo adhesion test was conducted on the animal models. In this case, the FRAPs maintained $60 \%$ bond strength after 5 weeks in vivo and in comparison to initial values (Figure 3g; Video S1, Supporting Information). Upon removal of the FRAPs, bone filaments were detected on the adhesives indicating a strong adhesion (Figure S13, Supporting Information).

\subsection{Evaluation of FRAP Fixation}

Finally, a direct comparison between gold standard techniques used in the clinics today and the FRAP methodology was sought out as a mean to cement the potential TEC adhesives for bone fixation surgeries. An assessment of fixation rigidity was performed where the FRAP was compared to LCP(TM) Compact Hand 1.5 (Depuy Synthes, West Chester, PA, US) (Compact Hand 1.5) or Kirschner wires (K-wires) using porcine metacarpal as fracture models (Figure 4). The porcine metacarpal was chosen due to the geometrical resemblance of human phalanges. Compact Hand 1.5, a stainless-steel plate, was placed over the fracture in a similar fashion as the FRAP and attached with eight screws into the bone in transverse fractures and six in oblique fractures. K-wires, $1.2 \mathrm{~mm}$ in diameter, were drilled through the bone longitudinally. Two crossed wires were used for each sample. To evaluate the fixation stability and estimate a fatigue resistance of the FRAP fixation, cyclic loads relevant to rehabilitation exercises were applied on the fracture models with transverse and oblique fractures. ${ }^{[18]}$ Loads between 10 and $70 \mathrm{~N}$ were applied continuously for 1000 cycles under humid conditions. The rigidity was calculated from the initial elastic region of the slope of the first load increase of the cyclic test. As shown in Figure 4a,b, the fixation with FRAP was far superior in rigidity compared to K-wires and matched the performance of Compact Hand 1.5 fixation in both transverse and oblique models. No difference in the disturbance of the fixation could be seen between Compact Hand 1.5 and FRAP fixation at the time period and loads used. Neither the movement during load cycles nor the maximum displacement during the test was significantly different (Table 1), which displays a firm fatigue resistance of the FRAP in the conditions used. However, the K-wires displayed both a large movement and displacement during the cyclic test, which corresponds to the low rigidity of the fixation. In this case, a twisting motion of the fractures occurred for the K-wire fixation, both in the transverse and oblique models, which led to movement of the specimen and finally failure of the test. From a recovery point of view, early mobilization is vital in phalangeal fractures as a mean to reduce finger stiffness complications. ${ }^{[19]} \mathrm{K}$-wire 

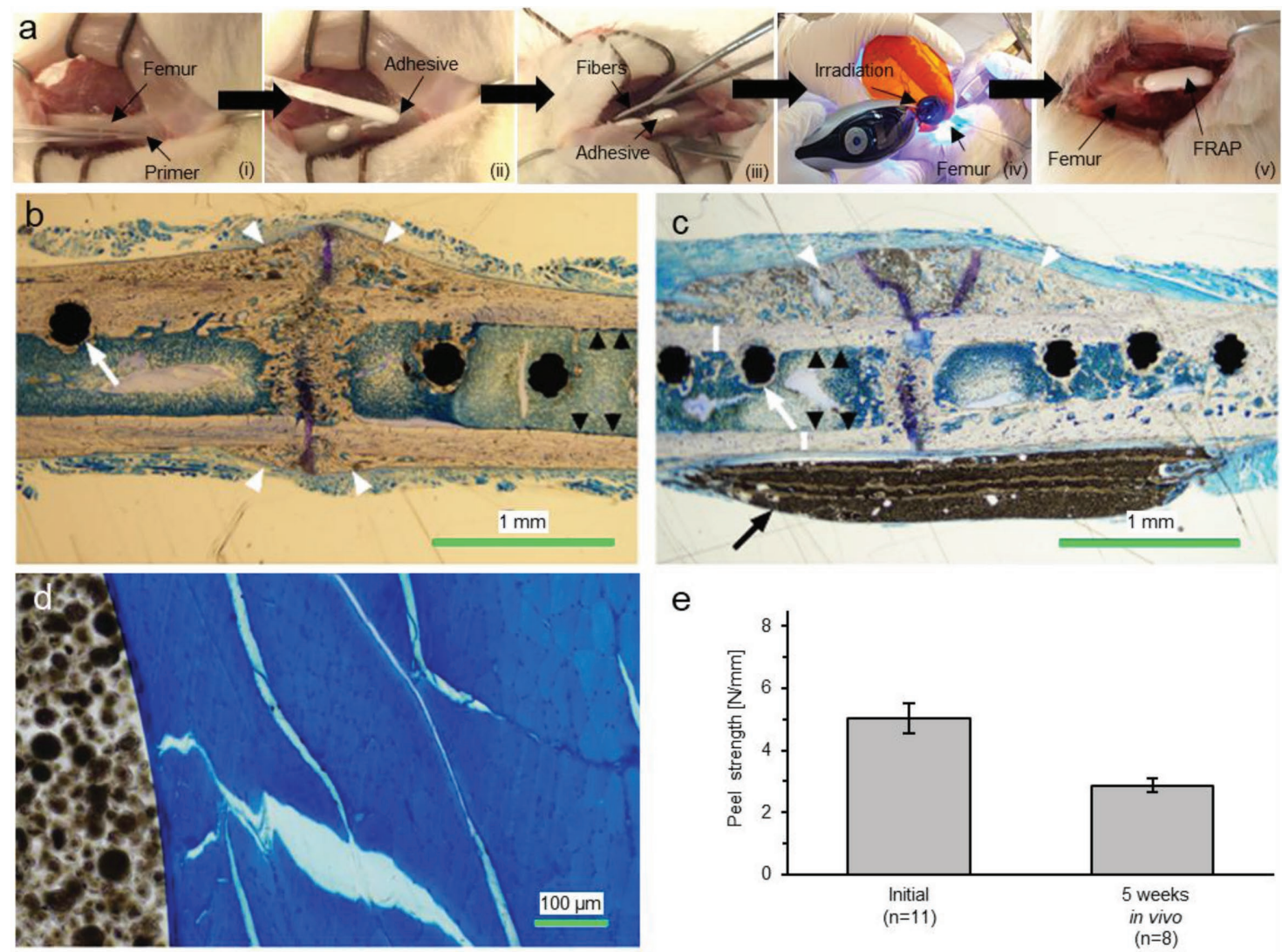

e

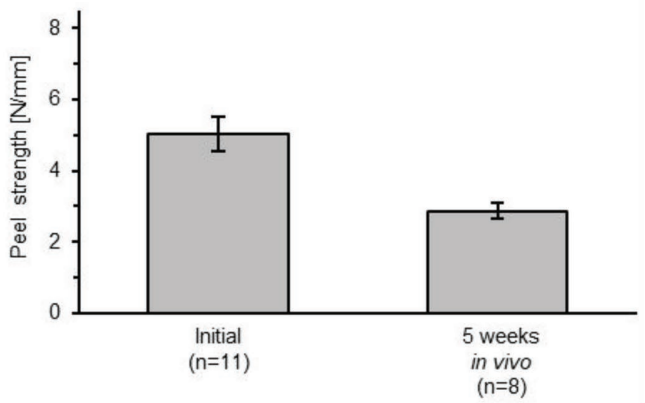

Figure 3. a) FRAP build-up on rat femur: i) primer application, ii) adhesive application, iii) fibers embedded to the adhesive, iv) curing the adhesive through light irradiation, and v) FRAP with four layers of adhesive and three layers of fiber on femur. b,c) Overview of healed femur bone fracture. White arrows indicate a screw from RatFix. White arrow heads indicate callus formation, black arrow heads and white lines indicate cortical bone, black arrow indicates FRAP. d). The musculature (blue) in connection to the FRAPs has a normal morphology without any inflammatory cells present. e) Peel bond strength of FRAP on rat femur, initial strength and strength after five weeks in vivo $(p<0.05)$. Staples in graphs represent average values, error bars are s.e.m. Two-sided Mann-Whitney U-test was used for statistical analysis.

fixations are generally too weak to allow immediate finger mobilization and plaster cast immobilization up to 4 weeks is common. The FRAP fixation provides a fracture fixation solution with similar fixation rigidity to Compact Hand 1.5 but superior to K-wires.

\section{Conclusions}

In summary, we herein introduce TEC chemistry as a powerful synthetic tool for creating biocompatible and high-performance adhesives suited for bone fracture fixation via fiber-reinforced adhesives patch methodology. A novel thiol-ene adhesive system was identified having the capacity to outperform commercially available dental composition by $55 \%$ and with respect to adhesive bond strength to wet bone. The extraordinary adhesive strength to bone surfaces was strongly dictated by a unique primer composition with controlled amount of phosphonic acid as well as the accessible enes for covalent linkages to the triazine-trione TEC adhesives. The FRAPs based on TEC adhesives encompass all the prerequisites as a future fixation methodology suited for bone fractures with high biocompatibility and in vivo functionality such as no cytotoxicity or genotoxicity, no inflammatory response, as well as no adverse effects on bone healing.
Finally, the FRAPs were found to withstand a fatigue test with loads between 10 and $70 \mathrm{~N}$ for 1000 cycles on porcine metacarpal fractures. Finally, the provided findings detail the FRAP methodology as a powerful adhesive alternative that match the current gold standard of fixation with plates and screws and biomechanically surpass fixation properties of K-wires, both clinically used in phalangeal and other low load bearing fractures.

\section{Experimental Section}

Materials Synthesis and Characterization: Synthesis and characterization details for the new primer components can be found in the Supporting Information.

Mechanical Evaluation of Adhesive Material: Beams of adhesive material with the dimensions of $1 \times 5 \times 35 \mathrm{~mm}$ were manufactured in poly(tetrafluoroethylene) molds. The adhesives were cured using light-emitting diode (LED) polymerization lamp (Bluephase 20, Ivoclar Vivadent AG, Liechtenstein), with wavelengths of 385-515 nm with a dominant wavelength of 400 and $470 \mathrm{~nm}$ and a maximum intensity of 2000-2200 mW cm-2 , intended for light-cured dental materials for $20 \mathrm{~s}$ per surface area of the adhesive. Mechanical performance was tested in threepoint bending using Instron 5566 (Instron Korea LLC) with a $500 \mathrm{~N}$ load cell and a center-center distance of the lower contacts was $30 \mathrm{~mm}$. The measurements were conducted at $23{ }^{\circ} \mathrm{C}$ at a relative humidity of $50 \%$. Data 

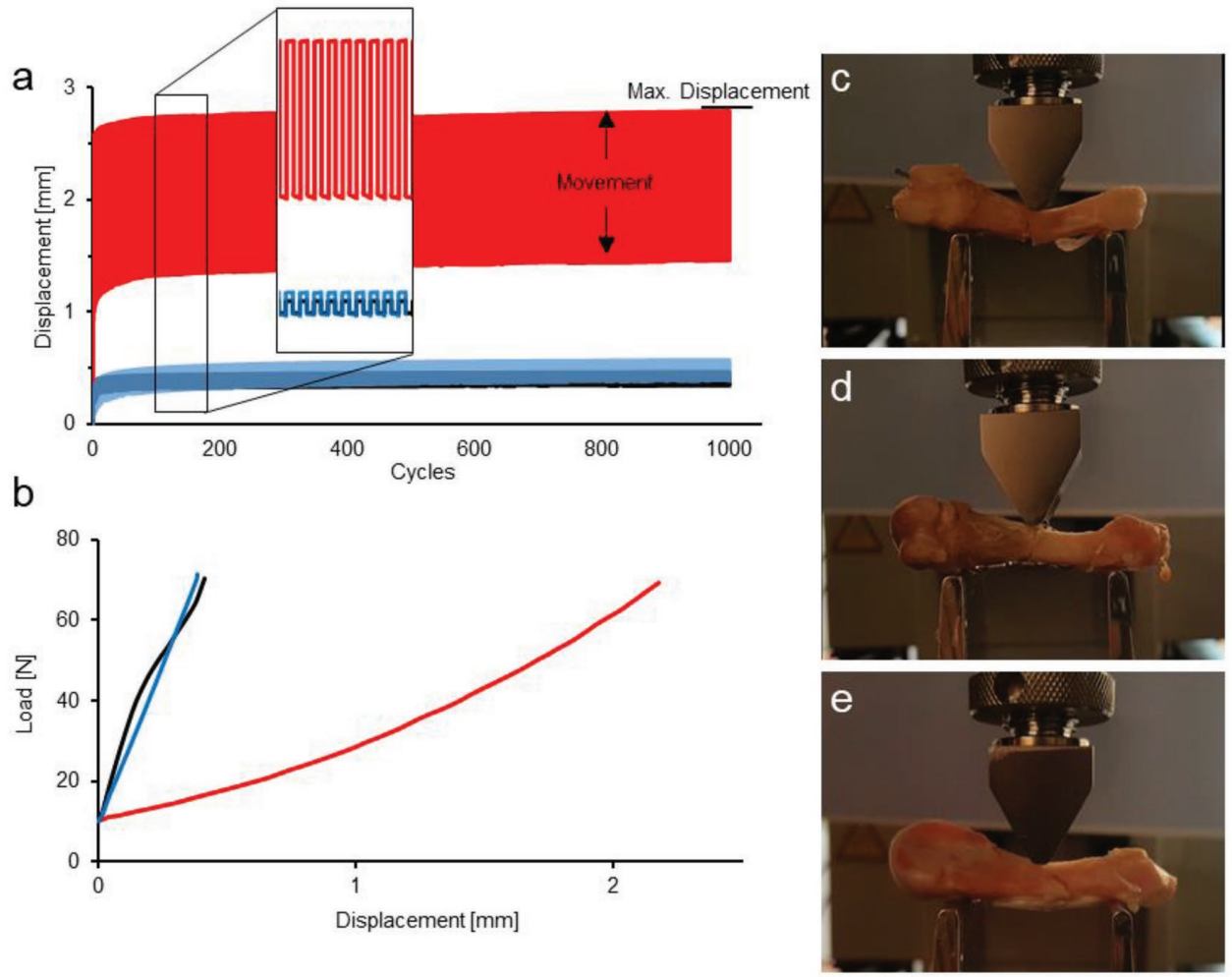

Figure 4. a) Displacement to cycle curve of cyclic three-point bending test of K-wire (red), Compact Hand 1.5 (black) and TEC FRAP (blue) fixations of a transverse fracture. b) Load and displacement curve of first load increase of the cyclic three-point bending test of K-wire (red), Compact Hand 1.5 (black) and TEC FRAP (blue) fixation of a transverse fracture. Images of the dislocation at maximum load of $70 \mathrm{~N}$ of c) K-wires with transverse fracture, d) Compact Hand 1.5 with oblique fracture, and e) TEC FRAP with transverse fracture.

were collected using Bluehill software. The flexural modulus was calculated through Equation (1), where $L$ is the distance between the lower contacts, $m$ is the slope at the initial elastic region of the load and displacement curve, $w$ is the width of the beam, and $d$ is the thickness of the beam. The test was performed once with five specimen of each adhesive. The twosided Mann-Whitney U-test was used for statistical analysis.

$E_{f}=\frac{L^{3} m}{4 w d^{3}}$

FT-Raman Analysis of Adhesive: FT-Raman spectroscopy was performed using a Perkin-Elmer Spectrum 2000 NIR FT-Raman instrument. The resin was analyzed with eight scans and the cured adhesive was analyzed with 32 scans with a laser power of $500 \mathrm{~mW}$. The spectra were normalized using the carbonyl shift $\left(1760 \mathrm{~cm}^{-1}\right.$, PerkinElmer Spectrum (10.5.1)). The shifts of thiol groups $\left(2575 \mathrm{~cm}^{-1}\right)$ and allyl groups $\left(1645 \mathrm{~cm}^{-1}\right)$ were analyzed to confirm the high conversion

Table 1. Results from three-point bending cyclic tests of fracture fixation of transverse and oblique fractures using K-wires, Compact Hand 1.5 or TEC FRAP.

\begin{tabular}{lcccc}
\hline Fixation & Fracture & $\begin{array}{c}\text { Bending rigidity } \\
{\left[\mathrm{N} \mathrm{m}^{2}\right](\text { s.e.m. })}\end{array}$ & $\begin{array}{c}\text { Movement } \\
{[\mathrm{mm}](\mathrm{s.e.m} .)}\end{array}$ & $\begin{array}{c}\text { Maximum displacement } \\
{[\mathrm{mm}](\mathrm{s.e.m} .)}\end{array}$ \\
\hline K-wires & Transverse & $0.02(0.01)$ & $1.3(0.1)$ & $3.1(0.5)$ \\
& Oblique & $0,014(0.002)$ & $1.0(0.2)$ & $2.9(0.2)$ \\
Compact Hand 1.5 & Transverse & $0.10(0.02)$ & $0.18(0.03)$ & $0.55(0.09)$ \\
& Oblique & $0.10(0.04)$ & $0.16(0.08)$ & $0.62(0.11)$ \\
TEC FRAP & Transverse & $0.083(0.013)$ & $0.13(0.01)$ & $0.62(0.09)$ \\
& Oblique & $0.10(0.01)$ & $0.16(0.01)$ & $0.53(0.05)$ \\
\hline
\end{tabular}

TEC reaction. Measurements were performed twice using two separate resin mixtures.

FRAP Shear Bond Strength: Rectangular bone pieces $(4 \times 1.5 \mathrm{~cm})$ were polished to obtain even surfaces and finally sanded using the P80 sand paper. The pieces were kept wet with $\mathrm{m}$-SBF. Fiber-reinforced adhesive patches were used to glue bone pieces together in pairs by bridging over the gap between the two bone pieces. Two patches per pair were used in a double-lap shear strength mode to form an even force distribution. The patches were built up by first wiping the wet bone surface with a tissue cloth and then spreading $4 \mu \mathrm{L} \mathrm{cm}{ }^{-2}$ of primer solution on the bonding area of each bone piece. The primer solution was dried with gentle air flow. Six consecutive layers of adhesive matrix surrounding five layers of surgical mesh were built up. Curing was induced using an LED polymerization lamp (Bluephase 20, Ivoclar Vivadent AG, Liechtenstein), with wavelengths of $385-515 \mathrm{~nm}$ with a dominant wavelength of 470 and $400 \mathrm{~nm}$ and a maximum intensity of $2000-2200 \mathrm{~mW} \mathrm{~cm} \mathrm{c}^{-2}$, intended for light-cured dental materials. The first layer was cured with irradiation for $20 \mathrm{~s}$ with TURBO mode $\left.(2000 \mathrm{~mW} \mathrm{~cm})^{-2}\right)$, and the rest of the layers were irradiated for $10 \mathrm{~s}$ with TURBO mode. As comparison, FRAPs with Histoacryl (B. Braun), Adper Scotchbond 1XT (3M), and Clearfil SE Bond (Kuaray) were made with curing procedures according to their manuals. The FRAPs were submerged in $\mathrm{m}$-SBF directly after final irradiation sequence and stored for $24 \mathrm{~h}$ at $37^{\circ} \mathrm{C}$ and then let to cool down to $23{ }^{\circ} \mathrm{C}$ before testing. For shear bond strength testing, an Instron 5566 instrument, Instron Korea LLC, with a $10 \mathrm{kN}$ load cell

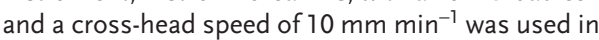
tensile mode. A preload of $1 \mathrm{~N}$ and a preload speed of $2 \mathrm{~mm} \mathrm{~min}^{-1}$ were used. The measurements were conducted at $23{ }^{\circ} \mathrm{C}$ at a relative humidity of $50 \%$. 
The specimens were kept wet as long as possible until they were attached to the machine. Three to six specimens were used for the tested samples. Data were collected using the Bluehill software. Adhesive bond shear strength was calculated from maximum load at failure divided by the area of failure.

Cytotoxicity Comparison of Adhesive Materials: An elution test was performed to compare in vitro toxicity of FRAP and selected commercialized materials, Histoacryl, Adper Scotchbond 1 XT, and Clearfil SE Bond. All the materials were cured on $1.5 \mathrm{~cm}^{2}$ poly(ethylene terephthalate) (PET) medical fiber and sterilized with two different methods: UV for $3 \mathrm{~h}$ or incubated in 2-propanol for $24 \mathrm{~h}$. Then dry samples incubated in $1 \mathrm{~mL}$ complete dulbecco's modified Eagel medium (DMEM) for $24 \mathrm{~h}$ to allow potential compounds leach out. Then, the testing medium were transferred into 96-well plate (Raw 264.7 or hDF, 5000 cells per well) with $100 \mu \mathrm{L}$ per well and incubated with another $72 \mathrm{~h}$. MTT test was applied for the viability evaluation and data were acquired with plate reader Infinite M200 (Tecan, Switzerland) at $570 \mathrm{~nm}$. For each sample, three parallel pieces were prepared to obtain leached-out medium for each cell line. And for each piece of material, six parallel wells were conducted in MTT assay.

In vivo Assessment of FRAP on Rat Femur: All animal experiments were performed after prior approval from the local ethics committee for animal studies for animal studies at the administrative court of appeals in Gothenburg, Sweden. Female Sprague-Dawley rats (Charles River, Germany) with an average weight of $250 \mathrm{~g}$ were used. The animals were acclimatized at least one week before surgery and housed with three to four rats in the same cage, with free access to food and water. A study was performed, where 38 animals had surgery of which 22 had femur fracture and 16 had intact femur. The rats with fractured femur where divided into two groups, 9 rats for which the femur was stabilized with Ratfix (RI system AG, Switzerland) only and 13 rats for which the femur was stabilized with Ratfix + FRAP. The rats with intact femur were divided into two groups, 12 rats were used for peel strength analysis and 4 rats were used for studies of potential inflammatory response. Anesthesia was induced by inhalation of isoflurane (Isobavet, SheringPlough Animal Health, Farum, Denmark) and pain relief achieved by subcutaneous injections of karprofen $\left(5 \mathrm{mg} \mathrm{kg}^{-1}\right.$, Rimadyl, Pfizer, Luxemborg) and buprenorfin (48 $\mu \mathrm{g} \mathrm{kg}^{-1}$, Temgesic, Shering-Plough, Brussels, Belgium). Postoperative additional doses of karprofen were given twice daily for $2 \mathrm{~d}$.

The right hind leg of the rat was shaved and disinfected with chlorhexidine. A longitudinal incision was placed on the femur just distal of the hip ending proximal of the knee joint. The fascia lata was opened and the muscle bluntly dissected. The femur was reached by splitting the musculus vastus lateralis and musculus biceps femoris. Blunt dissection was performed circumferentially, at the site where the osteotomy was planned.

For the rats where RatFix was used, six screws and a plastic plate were attached to the femur and a fractured was performed through the femur at the center of the RatFix using a Gigly wire saw, $\varnothing 0.2 \mathrm{~mm}$, according to the manufacturer's instructions. For the fractured femur rats where FRAP was used, the FRAP was added on top of the fractured area, $\approx 0.5 \mathrm{~cm}$ on each side of the fracture fixation and $\approx 90^{\circ}$ on the femur in relation to the RatFix. The FRAP fixation commenced with roughening of the bone surface with an abrasive pin, followed by washing and drying with wet and dry swab sticks. Primer was applied and dried with pressurized atmospheric air. Glue was applied, and on top of that, a layer of fiber was applied. The glue was cured by LED polymerization lamp (Bluephase 20i), with wavelengths of $385-515 \mathrm{~nm}$ with a dominant wavelength of 400 and $470 \mathrm{~nm}$ and a maximum intensity of 2000-2200 mW cm ${ }^{-2}$, intended for light-cured dental materials for $10 \mathrm{~s}$ per surface area of the adhesive, and this procedure was repeated three consecutive times. Finally, the surface of the FRAP was covered with a layer of glue and exposed photoinitiating light again. For the rats with intact femur, FRAP was applied in the same way as described above except for the fracture, with the difference that for 12 animals, that fibers used was $\approx 2 \mathrm{~cm}$ longer that the FRAP glue. The free parts of the fibers were later used for gripping by the biomechanical device when measuring the adhesiveness after euthanization. The muscle and fascia were sutured back in anatomical position in two layers using Vicryl 4/0 (Ethicon, Sweden) and the skin was sutured intradermally using Monocryl 5/0 (Ethicon, Sweden). After surgery, the rats were allowed to use full load on the operated limb. All surgeries were performed by one of the authors (J.v.K.) and all the FRAPS were applied by another author (J.P.). The surgeries were performed under sterile conditions. Directly after surgery, all animals were X-rayed to verify that the femur parts were located in position.

Five weeks after surgery, animals were euthanized with an overdose of pentobarbital (Alfatal, $100 \mathrm{mg} \mathrm{mL}^{-1}$, Omnidea AB, Stockholm). The rats were $\mathrm{X}$-rayed and the operated femurs were dissected out. For the 12 rats with intact femur and with longer FRAP fibers, the left hind leg was separated intact from the rat at the hip joint. FRAPs, with long fibers, were applied to these femurs and peel strength analysis was performed, to be compared with the corresponding right femur with FRAP exposed to in vivo conditions for 5 weeks.

In Vivo Adhesion Evaluation of FRAP: The application of FRAPs for peeling tests after 5 weeks in vivo is described above. As reference, FRAPs were applied in newly dissected rat femurs in the same way as they were applied in vivo. The femurs were attached to a Planar Biaxial TestBench Instrument (TA Instruments-ElectroForce System Group, Eden Prairie, MN, USA) and peeling test was conducted at room temperature and with a cross-head speed of $0.5 \mathrm{~mm} \mathrm{~s}^{-1}$. Time and displacement were recorded and force was measured by a $50 \mathrm{lbs}$ load cell using software WinTest 7.01 (TA Instruments-ElectroForce System Group, Eden Prairie, MN, USA). The maximum load and the width of the FRAP were used to calculate the peel strength.

Histological Evaluation of FRAP: Three femurs each from fractured femurs stabilized with RatFix only, RatFix/FRAP and intact femurs with FRAP were fixated in 4\% PFA, embedded in resin, and sectioned and stained with toloudine blue for analysis of inflammatory responses and bone healing characteristics.

Ex Vivo Fracture Fixation Evaluation of FRAP: The second and fifth metacarpal was dissected from porcine feet obtained from a butcher shop. The metacarpals were cleaned from soft tissue and a transverse or $45^{\circ}$ oblique fracture was cut through the bone pieces. The bone pieces were cleaned with water and the surface was roughened with a diamond abrasive pin and cleaned again with a wet tissue. $4 \mu \mathrm{L}$ of primer was applied per $\mathrm{cm}^{2}$ of the bone surface. The primer was dried with gentle airflow and then a drop of adhesive was applied on the primed surface, a $23 \times 7 \mathrm{~mm}$ fiber sheet was pressed down in the adhesive and the adhesive was then cured with irradiation using an LED polymerization lamp (Bluephase 20i), with wavelengths of 385-515 nm with a dominant wavelength of 470 and $400 \mathrm{~nm}$ and an intensity of $2000-2200 \mathrm{~mW} \mathrm{~cm}$, intended for light-cured dental materials. The total time of irradiation was $10 \mathrm{~s} \mathrm{~cm}^{-2}$. This procedure was done three times with a consequent shortening of the applied fiber sheets to 15 and $10 \mathrm{~mm}$ to achieve a patch with three fiber layers. Then a thin final layer of adhesive was applied followed by curing. The glued bone pieces were wrapped in tissue paper soaked in PEST solution to be kept wet but not induce bacterial growth. To do the three-point bending test the bone piece was attached to an Instron 5566 instrument, Instron Korea LLC, with a $500 \mathrm{~N}$ load cell and a cyclic force from 10 to $70 \mathrm{~N}$ was applied with a cross-head speed of $25 \mathrm{~mm} \mathrm{~min}{ }^{-1}$. A preload of $10 \mathrm{~N}$ and a preload speed of $5 \mathrm{~mm} \mathrm{~min} \mathrm{~mm}^{-1}$ were used. A span length between the lower supports of $3 \mathrm{~cm}$ was used. The measurements were conducted at $23{ }^{\circ} \mathrm{C}$ and a relative humidity of $50 \%$. The specimens were kept wet as long as possible until they were attached to the machine. Five specimens were used for the tested samples. Data were collected using the Bluehill software.

Scanning Electron Microscopy (SEM): SEM analysis of the FRAP crosssection was executed using a Hitachi Tabletop Microscope TM1000 using $15 \mathrm{kV}$ acceleration voltage. The samples were cryofractured by cooling the samples in liquid nitrogen before fracture.

Hydroxyapatite particles were imaged with Hitachi s4800 FE-SEM using $1 \mathrm{kV}$ acceleration voltage.

Four femurs and FRAP counterparts used in the adhesion tests were prepared and analyzed by SEM. Sample preparation consisted of primary fixation in $4 \%$ PFA, secondary fixation in $2 \%$ osmium 
tetroxide, dehydration in ethanol series (30-99.5\%), submersion in hexamethyldisilazane, air-drying, and finally sputter coating by anticharging metallic film (Au/Pd deposited by GATAN PECS Mod 681). SEM imaging was performed using Zeiss Supra 40VP field emission microscope operated at $4 \mathrm{kV}$ acceleration voltage in secondary electron detection mode. Analysis was done on the bone areas with intact surface and surface affected by FRAP application. Furthermore, the peeled-off FRAP was analyzed on the side which was in contact with bone.

$X$-Ray Microcomputed Tomography $(\mu \mathrm{CT})$ : $\mu \mathrm{CT}$ X-ray was performed on three different specimen types after five weeks in vivo; intact femurs without implant $(n=3)$, RatFix on fractured femur $(n=9)$ and RatFix/ FRAP on fractured femur $(n=13)$ stabilized femurs were selected for $\mu C T$ analysis. After the femurs were excised, they were kept moist in room temperature and analyzed postmortem within $8 \mathrm{~h}$ by $\mu \mathrm{CT}$ (SkyScan 1176, Bruker microCT, Kontich, Belgium), operating at $55 \mathrm{kV}, 453 \mathrm{~mA}$ with a $840 \mathrm{~ms}$ integration time and a pixel size of $8.82 \mu \mathrm{m}$, step size $0.42^{\circ} \mathrm{s}^{-1}$ through $197.4^{\circ}$ rotation and a $0.2 \mathrm{~mm}$ Al filter. Reconstruction, rendering and analysis were performed using the Skyscan software package (NRecon 1.6.9.8, Dataviewer 1.5.3, CTan 1.16.9.0). Furthermore, the volume of interest for each bone was defined as $1 \mathrm{~mm}$ proximal and distal to the induced fracture line and at similar position for the intact femurs. Semi-automatic segmentation was used to derive both the cortical bone region and the newly formed bone region (callus). These regions were analyzed separately with regard to bone mineral density (BMD), in $\mathrm{g} \mathrm{m}^{-3}$, of the newly formed bone, total bone volume (BV), in $\mathrm{m}^{3}$, bone volume fraction of tissue volume (BV/TV), mean crosssectional bone area, in $\mathrm{m}^{2}$.

Mechanical Evaluation of Bone Healing: After finalization of the $\mu \mathrm{CT}$. analysis, $\approx 12 \mathrm{~h}$ post mortem, femurs were subjected to three-point bending test (Planar Biaxial TestBench Instrument, TA InstrumentsElectroForce System Group, Eden Prairie, MN, USA), conducted at room temperature. 10 intact femurs were added to the 3 intact femurs subjected to $\mu \mathrm{CT}$-analysis, 9 out of 9 and 7 out of the 13 was tested for the RatFix and RatFix/FRAP stabilized group, respectively. All femurs were mounted in the anterior-posterior plane with the posterior surface of the bone resting on the two lower supports, $4 \mathrm{~mm}$ solid stainless-stee bars with a center-center distance of $16 \mathrm{~mm}$. The first support rested immediately proximal to the condyles whiles the second just distal to the lesser trochanter. The bones were preloaded to $5 \mathrm{~N}$ and allowed to adapt for $10 \mathrm{~s}$ and tested to fracture at a speed of $0.1 \mathrm{~mm} \mathrm{~s}^{-1}$ while time and displacement were recorded and force was measured by a $50 \mathrm{lbs}$ load cell using software WinTest 7.01 (TA Instruments-ElectroForce System Group, Eden Prairie, MN, USA). The force-displacement curve was used to determine the absorbed energy, the flexural stiffness, and the flexural strength. The mechanical data were normalized with the area of bone cross-section.

Cytotoxicity of Compounds Evaluated through In Vitro Cell Viability Tests: HDF cells were maintained in DMEM medium with $10 \%$ FBS and $100 \mathrm{U}$ penicillin-streptomycin solution. Cells were washed and harvest through trypsin and transferred into 96 well plates a density of 5000 cells per well and incubated for $24 \mathrm{~h}$ before use. LAP, IC819, TATATO, ETTMP, BAPA, and BAPA ${ }^{\text {bisphn }}$ were dissolved in EtOH and diluted in DMEM to prepare working medium with final concentrations $1 \times 10^{-6}, 10 \times 10^{-6}$, and $100 \times 10^{-6} \mathrm{M}$ of the compounds. Insoluble compounds HA and TEMPIC were incubated with $2 \mathrm{~mL}$ of DMEM medium for $24 \mathrm{~h}$ to prepare the elution medium. $1 \%, 10 \%$, and $100 \%$ of these elution media were used as the working medium. Old DMEM was removed from cells and replaced by fresh working medium above and incubated for another $24 \mathrm{~h}$. Then AlamarBlue tests were performed according to the instructions of the kit. Data were acquired with plate reader Infinite M200 (Tecan, Switzerland)) with fluorescent model ex/em wavelength at 560/590 nm. Data were acquired using i-control software. In all cases, 6 replicate wells were set for each sample and cells treated with PBS were used as negative control. Each test was repeated three times.

Cytotoxicity Evaluation of FRAP: A study to evaluate the cytotoxic potential of the FRAP was performed using FRAP extracts and cytotoxicity testing according to ISO 10993-5:2009 Annex C and RISE standard operating procedure SOP KM 11741. The FRAP was extracted at $37 \pm 1{ }^{\circ} \mathrm{C}$ for $24 \pm 2 \mathrm{~h}$ in Eagle's minimum essential medium (1x) with Earle's balanced salts solution (EBSS) buffered with $\mathrm{NaHCO}_{3}$ (Gibco Life Technologies) using a ratio of $3 \mathrm{~cm}^{2} \mathrm{~mL}^{-1}$. Extracts from FRAP, positive and negative controls as well as blanks (extraction vehicle not containing the test item but subjected to conditions identical to those to which the test item was subjected to during extraction) were added to a subconfluent monolayer of L929 mouse fibroblast cells and incubated for $24 \mathrm{~h}$ at $37 \pm 1{ }^{\circ} \mathrm{C}$ in $5 \pm 1 \% \mathrm{CO}_{2}$. Six specimens of each group were tested. After incubation, the extracts were removed and MTT (Sigma-Aldrich, cat. M5655) solution was added to the cells which were incubated for additional $2 \mathrm{~h}$ at $37 \pm 1{ }^{\circ} \mathrm{C}$ in $5 \pm 1 \% \mathrm{CO}_{2}$. Following incubation the MTT solution was removed, 2-propanol (Fischer Chemicals cat. P/7500/PB17) was added and the plates were shaken rapidly. Finally, the absorbance was measured at $570 \mathrm{~nm}$ (reference wavelength $650 \mathrm{~nm}$ ) and the viability of cells was calculated using BioTek Multi-Detection Microplate Reader Synergy 2 with dispense module. The software Gen5 Secure was used to control the plate reader and dispense module.

Genotoxicity Evaluation of FRAP: A Bacterial reverse mutation study based on the requirements of OECD guideline 471 (1997) in the ISO 10993 part 3 standard (2014), was performed by NAMSA under GLP conditions. The study was conducted to evaluate whether extracts of FRAP would cause mutagenic changes at the histidine locus of the Salmonella typhimurium tester strains TA98, TA100, TA1535, TA1537 or at the tryptophan locus of Escherichia coli tester strain WP2uvrA, in the presence and absence of mammalian metabolic activation. FRAP was extracted at $50 \pm 2{ }^{\circ} \mathrm{C}$ for $72 \pm 2 \mathrm{~h}$ in $0.9 \% \mathrm{NaCl}$ solution (Laboratoire Aguettant/Lavoisier, reference no. 600019) and at $37 \pm 1{ }^{\circ} \mathrm{C}$ for $72 \pm 2$ $h$ in $95 \% \mathrm{EtOH}$ (VWR PROLABO, ref. 20816.298), using a ratio of $3 \mathrm{~cm}^{2}$ $\mathrm{mL}^{-1}$. Tubes containing molten top agar were inoculated with culture from one of the five tester strains along with the test article extracts tested at the single dose of $100 \mu \mathrm{L}$ per plate of the $100 \%$ extract. All tester strains were from Trinova Biochem (Moltox), USA: S.typhimurium TA98 (Molten Part number 71-098L), S.typhimurium TA100 (Molten Part number 71-100L), S.typhimurium TA1535 (Molten Part number 71-1535L), S.typhimurium TA1537 (Molten Part number 71-1537L), E. coli WP2uvrA (Molten part number 72-188L). An aliquot of phosphate buffer or rat liver S9 mixture providing metabolic activation was added. The mixture was poured across triplicate plates. Parallel testing was conducted with negative control without FRAP extract and positive controls. 2-Nitrofluorene (Sigma-Aldrich, ref. N16754), 9-aminoacridine (Sigma-Aldrich, ref. A38401), methyl methanesulfonate (MMS) (Sigma-Aldrich ref. 129925), sodium azide (Sigma-Aldrich, ref S8032), 2-aminoanthracene (Sigma-Aldrich, ref. A38800) and benzo(a)pyrene (Sigma-Aldrich, ref. B1760) were used as positive controls. The mean number of revertants for the test extract plates was compared to the mean number of revertants of the appropriate negative control plates for each of the five tester strains.

Mouse Lymphoma Forward Gene Mutation Assay: A nonclinical GLP study was performed by NAMSA with the purpose of evaluating the mutagenic potential of FRAP extracts. The mouse lymphoma L5178Y/ $\mathrm{TK}^{+/-}$cell line (ATCC, CRL9518), heterozygous at the thymidine kinase (tk) locus, was used for this assay. FRAP was extracted $\left(3 \mathrm{~cm}^{2} \mathrm{~mL}^{-1}\right)$ in serum-free cell culture medium RPMIO (94.7\% v/v RPMI-1640 (Sigma-Aldrich, with sodium bicarbonate and $20 \times 10^{-3} \mathrm{M}$ HEPES), supplemented with $1 \% \mathrm{v} / \mathrm{v}$ L-glutamine, $1.8 \% \mathrm{v} / \mathrm{v}$ sodium pyruvate and $2 \% \mathrm{v} / \mathrm{v}$ penicillin-streptomycin (Sigma-Aldrich, ref. P44458-100ML) at $37^{\circ} \mathrm{C}$ for $72 \mathrm{~h}$ and in EtOH at $50^{\circ} \mathrm{C}$ for $72 \mathrm{~h}$, using a ratio of $3 \mathrm{~cm}^{2} \mathrm{~mL}^{-1}$. The RPMIO extract was supplemented with $5 \%$ inactivated horse serum (Sigma-Aldrich, ref H1138. Fischer Scientific, ref. 10368902) prior to 4 and $24 \mathrm{~h}$ treatments. The $\mathrm{EtOH}$ extract was diluted to a final concentration of $0.7 \%$ with RPMI5 for the 4 and $24 \mathrm{~h}$ treatments. The cells were exposed to the FRAP extracts and negative (extract vehicle alone) and positive controls of MMS and cyclophosphamide. The treatment was performed in duplicates for $4 \mathrm{~h}$ in the presence and absence of metabolic activation and $24 \mathrm{~h}$ in the absence of metabolic activation. Counting and sizing of colonies were performed to analyze the outcome of the tests. 
Mouse Peripheral Blood Micronucleus Study: A study was performed in adaptation to the methods described in the OECD (Mammalian erythrocyte micronucleus test, Guideline 474, 2014) and mentioned in the requirements of the ISO 10993 Standard: Biological evaluation of medical devices, Part 3 (2014): Tests for Genotoxicity, Carcinogenicity and Reproductive Toxicity: The tests was used to evaluate the potential for FRAP extracts to cause damage to chromosomes or the mitotic apparatus of murine erythroblasts by measuring the frequency of micronucleated reticulocytes of treated mice. The protocol has been approved by NAMSA Ethical Committee. FRAP was extracted in $0.9 \% \mathrm{NaCl}$ solution and sesame oil (Fluka) for $72 \pm 2 \mathrm{~h}$ (under agitations) at $50 \pm 2{ }^{\circ} \mathrm{C}$, using a $3.0 \mathrm{~cm}^{2}$ $\mathrm{mL}^{-1}$ ratio. Male and female mice (OF1 Ico (IOPS Caw), Charles River, France) $25-46 \mathrm{~g}$ were used. The mice were acclimatized for seven days before beginning of treatment. For three consecutive days, twelve mice per test article extract (male $n=6$ and female $n=6$ ) were injected at a dose of $20 \mathrm{~mL} \mathrm{~kg}^{-1}$ by intraperitoneal route for the $0.9 \% \mathrm{NaCl}$ extract and the sesame oil extract. In the same manner assessments with either the appropriate vehicle as the negative control or MMS as a positive control were conducted. All animals were observed immediately following dosing and daily for assessment of general health. On Day 4, blood was collected from the tail veins and reticulocytes of five samples/treatment group/sex were evaluated for the presence of micronuclei by flow cytometry.

Irritation Evaluation of FRAP: An evaluation of the irritating potential of FRAP was performed according to ISO TC 194/WG8 protocol "Evaluation of a method to detect skin irritation of medical device extracts using reconstructed human epidermis (RhE)," using the In Vitro EpiDerm TM Skin Irritation Test (EPI-200-SIT) kit according to the manufacturer's instructions. The FRAP was extracted in $0.9 \%$ saline (Sigma Aldrich) and in sesame oil (Fluka) for $72 \pm 2 \mathrm{~h}$ at $37 \pm 1{ }^{\circ} \mathrm{C}$ with continuous agitation with an extraction ratio of $3 \mathrm{~cm}^{2} / \mathrm{ml}$. Each extract was added to 3 individual tweresues. The tweresues were incubated for $18 \mathrm{~h}$ at $37 \pm 1{ }^{\circ} \mathrm{C}$ and $5 \pm 1 \% \mathrm{CO}_{2}$. Following incubation the extracts were removed by washing with DPBS (MatTek In Vitro Life Science Laboratories). The skin models were transferred into new 6-well plates prefilled with assay medium. After washing with DPBS, the tweresues were transferred to a 24-well plate containing MTT solution and incubated for $3 \mathrm{~h}$ at $37{ }^{\circ} \mathrm{C}$ in $5 \% \mathrm{CO}_{2}$. The MTT solution was removed and the tweresues were transferred to a 24-well plate pre-filed with 2-propanol. The tweresues were completely immersed in the 2-propanol and the plates were sealed to inhibit evaporation. The plates were shaken at $120 \mathrm{rpm}$ for $2 \mathrm{~h}$ to allow complete extraction of formazan from the cells. The inserts were pierced to allow the extract to run into the well where the tweresue was placed. $200 \mu \mathrm{L}$ of the solution was pipetted in duplicates into a 96-well plate and the absorbance was measured at $570 \mathrm{~nm}$.

\section{Supporting Information}

Supporting Information is available from the Wiley Online Library or from the author.

\section{Acknowledgements}

This work was generously supported by the Swedish Governmental Agency for Innovation Systems - Vinnova (2014-03777). M.M. thanks the Swedish Research Council VR (2010-435) and the Knut and Alice Wallenberg Foundation - KAW (2012-0196). S.G.G. thanks Marie Skłodowska-Curie actions (MSCA-IF-2014-655649) for funding.

\section{Conflict of Interest}

The authors declare no conflict of interest.

\section{Keywords}

adhesives, biomedical applications, composites, photochemistry, polymeric materials
Received: January 16, 2018

Revised: March 2, 2018

Published online:

[1] a) C. E. Hoyle, C. N. Bowman, Angew. Chem., Int. Ed. 2010, 49, 1540; b) A. B. Lowe, Polym. Chem 2014, 5, 4820.

[2] T. M. O'Shea, A. A. Aimetti, E. Kim, V. Yesilyurt, R. Langer, Adv. Mater. 2015, 27, 65

[3] a) J. A. Carioscia, H. Lu, J. W. Stanbury, C. N. Bowman, Dent. Mater. 2005, 21, 1137; b) H. Lu, J. A. Carioscia, J. W. Stansbury, C. N. Bowman, Dent. Mater. 2005, 21, 1129; c) M. Podgorski, E. Becka, M. Claudino, A. Flores, P. K. Shah, J. W. Stansbury, C. N. Bowman, Dent. Mater. 2015, 31, 1255; d) M. Podgorski, E. Becka, M. Claudino, A. Flores, P. K. Shah, J. W. Stansbury, C. N. Bowman, Dent. Mater. 2015, 31, 1263; e) S. Reinelt, M. Tabatabai, U. K. Fischer, N. Moszner, A. Utterodt, H. Ritter, Beilstein J. Org. Chem. 2014, 10, 1733; f) S. Reinelt, M. Tabatabai, N. Moszner, U. K. Fischer, A. Utterodt, H. Ritter, Macromol. Chem. Phys. 2014, 215, 1415.

[4] a) V. Granskog, O. C. J. Andren, Y. K. Cai, M. Gonzalez-Granillo, L. Fellander-Tsai, H. von Holst, L. A. Haldosen, M. Malkoch, Adv. Funct. Mater. 2015, 25, 6596; b) A. Nordberg, P. Antoni, M. I. Montanez, A. Hult, H. Von Holst, M. Malkoch, Acs Appl. Mater. Interfaces 2010, 2, 654; c) Y. Hed, K. Oberg, S. Berg, A. Nordberg, H. von Holst, M. Malkoch, J. Mater. Chem. B 2013, 1, 6015 ; d) K. Olofsson, V. Granskog, Y. Cai, A. Hult, M. Malkoch, RSC Adv. 2016, 6, 26398.

[5] a) G. M. Brauer, J. W. Kumpula, D. J. Termini, K. M. Davidson, J. Biomed. Mater. Res. 1979, 13, 593; b) I. Kilpikari, M. Lapinsuo, P. Tormala, H. Patiala, P. Rokkanen, J. Biomed. Mater. Res. 1986, 20, 1095; c) R. A. Chivers, R. G. Wolowacz, Int. J. Adhes. Adhes. 1997, 17, 127; d) M. C. Harper, M. Ralston, J. Biomed. Mater. Res. 1983, 17, 167; e) N. L. Hampel, G. J. Pijanowski, R. G. Johnson, Am. J. Vet. Res. 1986, 47, 1605; f) M. A. Akcal, O. Poyanli, K. Unay, I. Esenkaya, B. Gokcen, A. S. Firatligil, J. Orthop. Surg. Res. 2014, 9, 76.

[6] a) S. M. R. Tuusa, L. V. J. Lassila, J. P. Matinlinna, M. J. Peltola, P. K. Vallittu, J. Biomed. Mater. Res. B 2005, 75B, 334; b) K. Ishihara, N. Nakabayashi, J. Biomed. Mater. Res. 1989, 23, 1475; c) J. Vainio, J. Kilpikari, P. Tormala, P. Rokkanen, Arch. Orthop. Trauma Surg. 1979, 94, 191.

[7] a) G. di Nuzzo, M. Luongo, C. Parlato, A. Moraci, J. Craniofacial Surg. 2010, 21, 1170; b) P. W. M. Fedak, E. Kolb, G. Borsato, D. E. C. Frohlich, A. Kasatkin, K. Narine, N. Akkarapaka, K. M. King, Ann. Thorac. Surg. 2010, 90, 979.

[8] a) B. D. Winslow, H. Shao, R. J. Stewart, P. A. Tresco, Biomaterials 2010, 31, 9373; b) H. Zhang, L. Bre, T. Y. Zhao, B. Newland, M. Da Costa, W. X. Wang, J. Mater. Chem. B 2014, 2, 4067.

[9] a) A. Ekelund, O. S. Nilsson, Int. Orthop. 1991, 15, 331; b) G. Ciapetti, S. Stea, E. Cenni, A. Sudanese, D. Marraro, A. Toni, A. Pizzoferrato, Biomaterials 1994, 15, 63; c) G. Ciapetti, S. Stea, E. Cenni, A. Sudanese, D. Marraro, A. Toni, A. Pizzoferrato, Biomaterials 1994, 15, 92; d) G. Schneider, K. Otto, Eur. Arch. OtoRhino-Laryngol. 2012, 269, 1783; e) S. Szep, A. Kunkel, K. Ronge, D. Heidemann, J. Biomed. Mater. Res. 2002, 63, 53; f) N. H. Kong, T. Jiang, Z. C. Zhou, J. L. Fu, Dent. Mater. 2009, 25, 1371; g) E. G. Garza, A. Wadajkar, C. Ahn, Q. Zhu, L. A. Opperman, L. L. Bellinger, K. T. Nguyen, T. Komabayashi, J. Oral Sci. 2012, 54, 213; h) O. E. Dahl, L. J. Garvik, T. Lyberg, Acta Orthop. Scand. 1994, 65,147 ; i) R. Vaishya, M. Chauhan, A. Vaish, J. Clin. Orthop. Trauma 2013, 4, 157.

[10] a) M. Donkerwolcke, F. Burny, D. Muster, Biomaterials 1998, 19, 1461; b) C. Heiss, R. Kraus, D. Schluckebier, A.-C. Stiller, S. Wenisch, R. Schnettler, Eur. J. Trauma 2006, 32, 141; c) D. F. Farrar, Int. J. Adhes. Adhes. 2012, 33, 89; d) A. P. Duarte, 
J. F. Coelho, J. C. Bordado, M. T. Cidade, M. H. Gil, Prog. Polym. Sci. 2012, 37, 1031; e) V. Bhagat, M. L. Becker, Biomacromolecules 2017, $18,3009$.

[11] a) K. Ikemura, F. R. Tay, T. Hironaka, T. Endo, D. H. Pashley, Dent. Mater. 2003, 19, 707; b) Y. Yoshida, K. Nagakane, R. Fukuda, Y. Nakayama, M. Okazaki, H. Shintani, S. Inoue, Y. Tagawa, K. Suzuki, J. De Munck, B. Van Meerbeek, J. Dent. Res. 2004, 83, 454; c) K. Ikemura, F. R. Tay, N. Nishiyama, D. H. Pashley, T. Endo, Dent. Mater. J. 2006, 25, 566; d) K. Ikemura, F. R. Tay, N. Nishiyama, D. H. Pashley, T. Endo, Dent. Mater. J. 2007, 26, 105; e) D. Fukegawa, S. Hayakawa, Y. Yoshida, K. Suzuki, A. Osaka, B. Van Meerbeek, J. Dent. Res. 2006, 85, 941; f) Y. Yoshida, B. Van Meerbeek, Y. Nakayama, M. Yoshioka, J. Snauwaert, Y. Abe, P. Lambrechts, G. Vanherle, M. Okazaki, J. Dent. Res. 2001, 80, 1565 .

[12] a) I. C. C. M. Porto, D. C. Oliveira, R. A. Raele, K. H. S. Ribas, M. A. J. R. Montes, C. M. M. B. De Castro, Dent. Mater. 2011, 27, 221; b) Z. Z. Liu, Y. Tang, T. Kang, M. Y. Rao, K. J. Li, Q. Wang, C. Y. Quan, C. Zhang, Q. Jiang, H. Y. Shen, Colloid Surf. B 2015, 131, 39.

[13] E. Testai, M. Epstein, I. Emri, P. Hartemann, P. Hoet, N. Leitgeb, L. Martínez Martinez, A. Proykova, L. Rizzo,
E. Rodriguez-Farré, L. Rushton, K. Rydzynski, T. Samaras, E. Testai, T. Vermeire, P. Hartemann, E. Rodríguez-Farre, S. C. Rastogi, J. Bustos, U. Gundert-Remy, A. Hensten, H. M. Kopperud, N. Olea, A. Piersma, W. De Jong, Regul. Toxicol. Pharmacol. 2016, 79, 106.

[14] a) B. D. Fairbanks, M. P. Schwartz, C. N. Bowman, K. S. Anseth, Biomaterials 2009, 30, 6702; b) S. B. Anderson, C. C. Lin, D. V. Kuntzler, K. S. Anseth, Biomaterials 2011, 32, 3564.

[15] A. Oyane, K. Onuma, A. Ito, H. M. Kim, T. Kokubo, T. Nakamura, J. Biomed. Mater. Res. A 2003, 64a, 339.

[16] a) V. Besse, M. A. Derbanne, T. N. Pham, W. D. Cook, L. Le Pluart, Dent. Mater. 2016, 32, 561; b) V. Besse, L. Le Pluart, W. D. Cook, T. N. Pham, P. J. Madec, J. Polym. Sci., Part A: Polym. Chem. 2013, 51, 149.

[17] a) Z. Cherkaoui, P. Esfandiari, R. Frantz, J. J. Lagref, R. Liska, WO2012126695A1, 2012; b) I. Arslan-Alaton, S. Shayin, T. OlmezHanci, Environ. Technol. 2012, 33, 419.

[18] S. Edsfeldt, D. Rempel, K. Kursa, E. Diao, L. Lattanza, J. Hand Surg. Eur. Vol. 2015, 40, 705.

[19] D. Haughton, D. Jordan, M. Malahias, S. Hindocha, W. Khan, Open Orthop. J. 2012, 6, 43. 